\title{
AUTOPHAGY; Recent Advances in Health and Disease
}

\author{
AReview \\ Sahar Gamal Abo ElFadl \\ Article \\ Department of Medical Histology \& Cell Biology, Faculty of Medicine, Cairo University
}

\begin{abstract}
Macroautophagy (autophagy) meaning self-eating is an evolutionary conserved intracellular degradation pathway, during which autophagosomes envelop bulk cytosol, unwanted or damaged organelles and misfolded proteins to handle them to the lysosomes for breakdown. There are many types of autophagy, which differ mainly in the site of cargo sequestration and in the type of cargo itself. The process of autophagy is a model in which autophagic vesicles develop into mature degradative autophagolysosomes in a series of distinguished steps namely: initiation, nucleation, expansion, maturation then finally degradation. The discovery of definitive biological markers for autophagy by the Japanese, Nobel Prize-awarded cell biologist Yoshinori Ohsumi and the advances in visualizing techniques enabled further insight of this vital process. Autophagy takes place at a low basal level constitutively, and can be potently induced by various types of stress conditions, such as starvation, hypoxia, pathogen invasion, and exercise. The functional relationship between apoptosis and autophagy is complex. Under certain circumstances, autophagy constitutes a stress adaptation that avoids cell death (and suppresses apoptosis), whereas in other cellular events, it constitutes an alternative cell-death pathway. Recently, autophagy dysfunction is linked to severe diseases such as neurodegeneration and cancer. Control of autophagy promises to facilitate the development of therapeutic and preventive measures for these morbid diseases for the well-being of mankind.
\end{abstract}

Key Words: Apoptosis, autophagy, cancer, degenerative diseases, fasting.

Revised: 04 October 2018, Accepted: 13 October 2018

Corresponding Author: Sahar Gamal Abo ElFadl, Department of Medical Histology \& Cell Biology, Faculty of Medicine, Cairo University, Tel.: +20 1125285784, Fax:+202 35381183 / +202 35381760, E-mail: saharmg6161@gmail.com.

ISSN: 1110-0559, Vol. 2, No. 1

\section{HISTORICAL BACKGROUND}

Christian de Duve and his team discovered and characterized lysosomes in $1955^{(1)}$ then defined peroxisomes in $1965^{(2)}$. The so-called "dense bodies" were identified during attempts to purify lysosomes. They were charaterized as compartments similar to, but distinct from, lysosomes and they were named autophagic vacuoles (autophagosomes) also by De Duve in 1962 . One of the first clues to their degradative capacity was the detection of dense, amorphous material and even whole organelles within these vacuoles ${ }^{(3)}$.

In the early 1990's, almost 30 years after de Duve came up with the term autophagy, the process was still a biological ambiguity. Molecular markers were not available yet and ultimately components of the autophagy machinery were out of reach. Many fundamental questions remained unanswered: How was the autophagy process launched? How was the autophagosome established? How important was autophagy for cellular well being? Did autophagy have any impact in human disease pathogenesis?

In 1992 a Japanese cell biologist, Yoshinori Ohsumi, and his colleagues discovered that fortunately autophagy takes place in yeast. Using light microscopy, they noticed a few hours after starving protease-deficient yeast mutants of nutrients, that the vacuole (which functions like mammalian lysosome) was filled with vesicles containing parts of cytoplasm ${ }^{(4)}$. This has triggered studying the molecular biology of the autophagic machinery and identifying the participating key proteins. In an article published in FEBS Letters in 1993, Ohsumi reported his discovery of as many as 15 genes that are critical for the activation of autophagy in eukaryotic cells ${ }^{(5)}$.

Ohsumi and his team were the first to identify mammalian homologues of the yeast ATG genes, which allowed studies on the function of autophagy in higher eukaryotes. Shortly, genetic studies revealed that mice lacking the $\operatorname{Atg} 5$ gene are apparently normal at birth, but die during the first day of life due to inability to cope with the starvation that precedes feeding ${ }^{(6)}$. Studies of knockout mouse models lacking different components of the autophagy machinery have confirmed the importance of the process in a variety of mammalian tissues ${ }^{(7)}$.

An important first remark into the role of autophagy in disease came from the observation that Beclin-1, the product of the BECN1 gene, is mutated in a large proportion of human breast and ovarian cancers. BECN1 is a homolog of yeast ATG6 that regulates steps in the initiation of autophagy. This finding generated growing interest in the role of autophagy in cancer initiation ${ }^{(8)}$. 
After the persistent hard work for three decades, the 2016 Nobel Prize in Physiology or Medicine was duly awarded to Yoshinori Ohsumi for his discoveries of mechanisms for autophagy that paved the way to other scientists to explore furtherly this field ${ }^{(9)}$.

\section{INTRODUCTION}

Macroautophagy (autophagy) meaning self-eating is an evolutionary conserved intracellular degradation pathway, during which autophagosomes envelop bulk cytosol, unwanted or damaged organelles and misfolded proteins to handle them to the lysosomes for breakdown. The process is a major component in mammalian cell metabolism, homeostasis, differentiation and development, tissue remodeling, survival, in addition to protein and organelle quality control ${ }^{(10)}$. Autophagy basically enables cells to survive stress from the external environment, such as nutrient deprivation, as well as internal stresses like accumulation of damaged organelles and pathogen invasion ${ }^{(11)}$.

Autophagy is characterized by formation of autophagosomes, which are intracytoplasmic organelles enclosed by two or more membranes. Autophagosomes engulf damaged materials and degrade them. They dock with lysosomes, and the resulting autolysosome utilizes lysosomal enzymes to digest the contents of the autophagic vacuoles ${ }^{(12)}$.

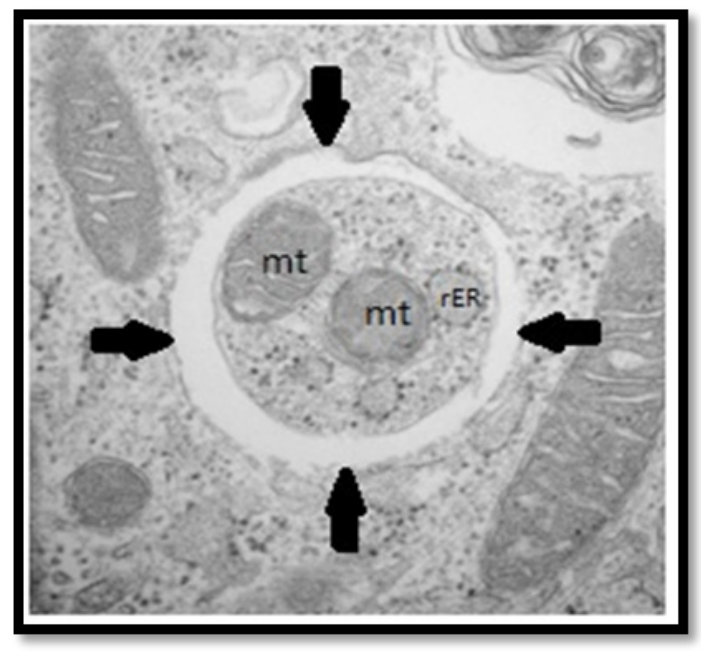

Fig. (1): Transmission electron micrograph showing a double membrane autophagosome (arrows) sequestering mitochondria (mt), rough endoplasmic reticulum (rER) and small amount of cytoplasm ${ }^{(13)}$.

In addition, autophagy has been recently addressed as an important cellular activity for protecting stem cells from damage by intrinsic or extrinsic factors during aging, tissue regeneration, and cellular reprogramming ${ }^{(14 \& 15)}$. As many normal types of cells require a certain well-controlled level of autophagy, any condition exceeding the capability of a cell to control can trigger a specific killing machinery: autophagic cell death $^{(16)}$.

\section{TYPES OF AUTOPHAGY}

There are many types of autophagy, which differ mainly in the site of cargo sequestration and in the type of cargo itself ${ }^{(17)}$.

Considering the site of cargo sequestration, the following types can be found:

1. Macroautophagy: cytoplasm is sequestered nonspecifically within cytosolic vesicles to be degraded in the lysosome releasing the resulting macromolecules back into the cytosol for reuse.

2. Microautophagy: involves direct uptake at the surface of the lysosome/vacuole, where the limiting membrane sequesters cytoplasm by invagination, protrusion and/or septation.

3. Chaperone-mediated autophagy: involves direct translocation of unfolded proteins across the lysosome membrane through receptor-driven degradative pathway the action of the cytosolic and lysosomal chaperone hsc70, and the lysosomal membrane protein LAMP-2A

4. Micro- and macropexophagy: specific autophagic removal of peroxisomes,

5. Piecemeal microautophagy: of the nucleus which is a specific type of autophagy

6. The cytoplasm-to-vacuole targeting (Cvt) pathway: it is a biosynthetic method of delivery for at least 2 vacuolar hydrolases.

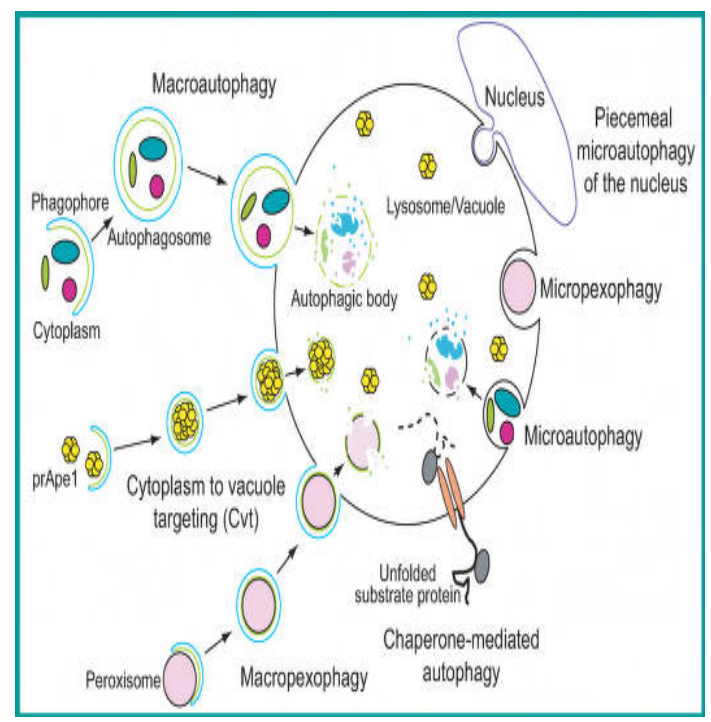

Fig. (2): A Diagram representing the different types of autophagy according to the site of cargo sequestration ${ }^{(18)}$.

According to the type of cargo inside the autophagosome the following different forms of autophagy can be recognized as:

1. "In bulk" Autophagy: contain proteins and organelles.

2. Mitophagy: contain mitochondria.

3. Ribophagy: contain ribosomes.

4. Pexophagy: contain peroxisomes. 
5. Endophagy: contain endoplasmic reticulum.

6. Lipophagy: contain lipid droplets.

7. Glycophagy: contain glycogen granules.

8. Aggrephagy: contain protein aggregates.

9. Allophagy: contain spermatozoid-inherited organelles after fertilization.

10. Xenophagy: contain intracellular pathogen.

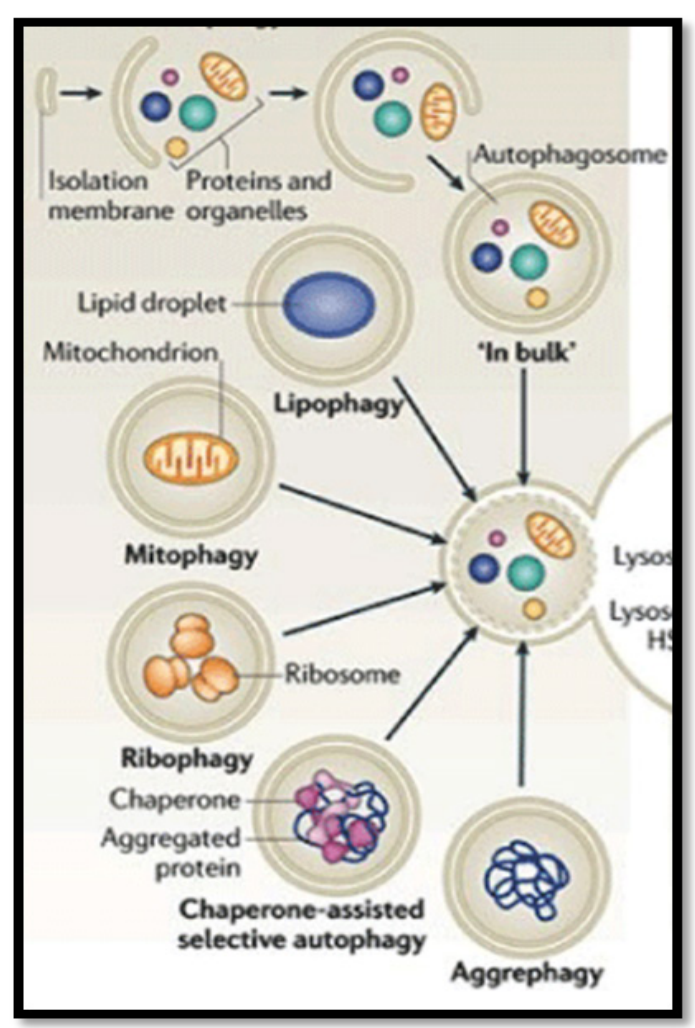

Fig. (3): Forms of autophagy according to cargo type ${ }^{(19)}$

At present, it is known that autophagy occurs at a basal level mainly for energy supply. On the other hand, in many cell types it is also an inducible process. So the control of autophagic induction is important for defense against extracellular pathogens as well. Although autophagy is generally considered to be nonspecific process, yet there are various examples of specific types of autophagy ${ }^{(8)}$.

\section{MECHANISM OF AUTOPHAGY}

The process of autophagy is a model in which AVs develop into mature degradative autophagolysosomes in a series of distinguished steps ${ }^{(20-21)}$ as follows:

1. Initiation of autophagy: it is achieved by formation of a supramolecular protein complex resulting from the linkage of many Atg1 complexes (dimers of pentameres) to each other by $A \operatorname{tg} 13$ as illustrated in fig. 4(A).

2. Nucleation: Initial isolation membrane (phagophore) will be formed facing the cargo to be degraded.

3. Expansion: where this membrane expands and elongates and the two ends merge into an AV [fig. 4(B)].

4. Maturation: Amphisome formation: resulting from fusion of the AV and an endosome or multivesicular body. The amphisome then acidifies to activate the hydrolytic enzymes after fusion with the lysosome in the next step.

5. Degradation: Fusion with lysosome where hydrolases start the breakdown of the inner membrane of the autophagosome together with the contents and the resulting molecules will diffuse back to the cytoplasm for further use [fig. 4(C)].
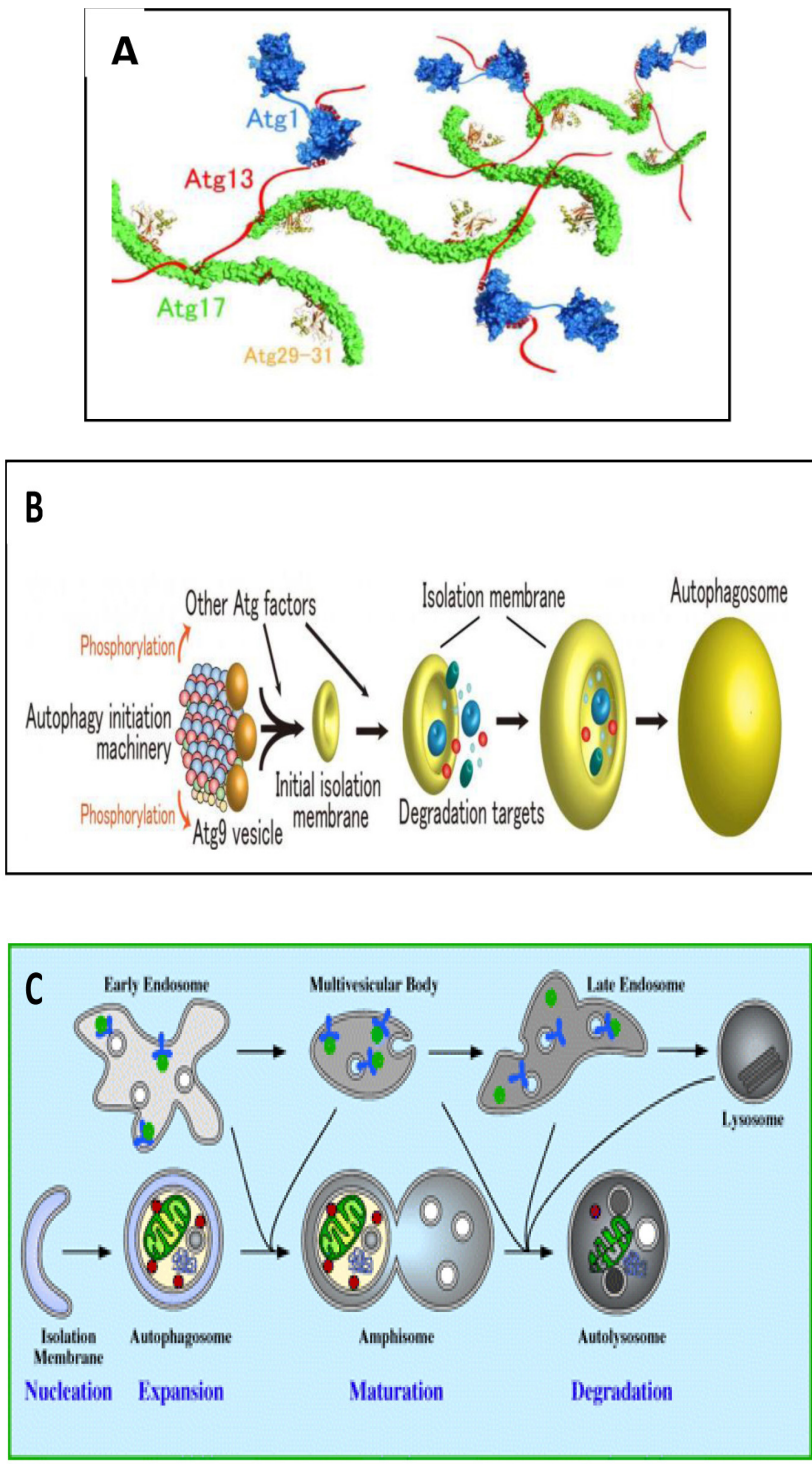

Fig. (4): Stages of autophagy. A) Initiation complex formation: Atg13 (red) links Atg1 (blue) and two Atg17s (green) to each other, thereby promoting formation of the giant complex. (B) Formation of the initial isolation membrane through the recruitment of Atg9 vesicles and Atg factors including Atg9. (C) Expansion and maturation of the autophagosome followed by fusion with the lysosome for degradation ${ }^{(22)}$.

One ambiguity that remained unsolved for decades was which membrane in the cell gives rise to phagophores??? Early studies provided evidence supporting both the Golgi 
and the endoplasmic reticulum (ER), as well as an area of the cell termed GERL (Golgi endoplasmic reticulum lysosomes), to be the source of the AV membrane ${ }^{(23)}$. More recent studies indicated that autophagic vesicles might be formed de novo through nucleation, assembly and elongation of small membrane structures ${ }^{(24)}$. However, in 2010 another team used fluorescently-labeled proteins to study the origin of phagophores. They observed that the outer mitochondrial membrane was the membrane source with some contribution from the endoplasmic reticulum ${ }^{(25)}$. Currently it is generally accepted to be derived from the endoplasmic reticulum (ER) in a specialized region known as the omegasome ${ }^{(26)}$ as illustrated in the following diagram (Fig. 5).

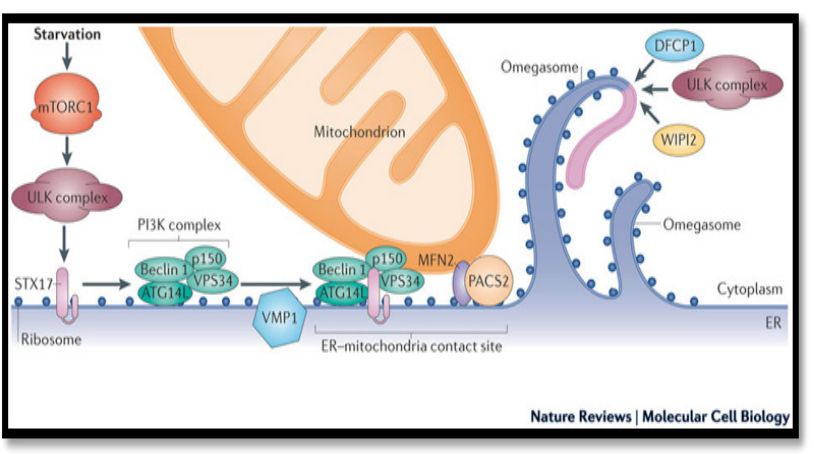

Fig. (5): A diagram showing the "Omegasome" which is the mostly accepted origin of the initial membrane ${ }^{(27)}$.

\section{AUTOPHAGY VERSUS APOPTOSIS}

Since 1972 when Kerr and his associates ${ }^{(28)}$ published their valuable work and discussed the term 'apoptosis' which has long been used as a synonym for programmed cell death. In their article, the authors classified cell death into two groups: apoptosis, signified the genetically controlled programmed cell death, and necrosis, described the passive or accidental type of cell death. However, in 1990 Clarke $^{(29)}$ furtherly highlighted that there are two types of programmed cell death in mammalian cells namely: apoptotic and autophagic.

Apoptotic cell death is proceeded by cell shrinkage, chromatin condensation, nucleosomal DNA degradation and fragmentation of the cell into so-called 'apoptotic bodies'. These characteristic morphological features result from activation of the caspase family of cysteine proteases. The remnants of the cell are removed by the lysosomes of professional phagocytes or neighboring cells after heterophagocytosis. On the other hand, autophagic cell death is characterized by the appearance of doubleor multiple-membrane cytoplasmic vesicles engulfing bulk cytoplasm and/or cytoplasmic organelles such as mitochondria and endoplasmic reticulum. Autophagic vesicles and their contents are destroyed by the lysosomal system of the same cell ${ }^{(30)}$.

The functional relationship between apoptosis and autophagy is complex in the sense that, under certain circumstances, autophagy constitutes a stress adaptation that avoids cell death (and suppresses apoptosis), whereas in other cellular settings, it constitutes an alternative celldeath pathway. Moreover, autophagy and apoptosis may be triggered by common upstream signals, and sometimes this results in combined autophagy and apoptosis; in other instances, the cell switches between the two responses in a mutually exclusive manner. On a molecular level, this means that the apoptotic and autophagic response machineries share common pathways that either link or polarize the cellular responses ${ }^{(31)}$.

\begin{tabular}{llll} 
ASSESSMENT & AND & VISUALIZATION & OF \\
\hline AUTOPHAGY & & &
\end{tabular}

1. Transmission Electron Microscopy: Autophagy was originally described using electron microscopy more than 50 years ago, when electron microscopy and sample preparation methods for biological materials had just emerged ${ }^{(32)}$. Despite the enormous developments in different methods for the monitoring of autophagy in cells and animals, transmission electron microscopy is still needed and can give both qualitative and quantitative information. The autophagosome is recognizable by transmission electron microscopy because of its characteristic double-walled membrane.

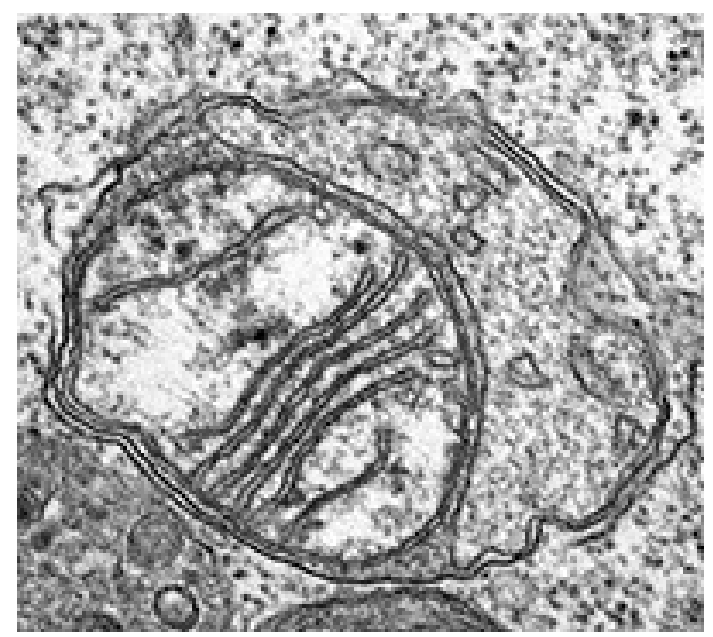

Fig. (6): An electron micrograph showing a mitochondrion inside a double membrane autophagosome (Mitophagy) ${ }^{(33)}$.

However, interpreting electron microscopic data is subjective, especially distinguishing autophagosomes and autolysosomes, and even differentiating autophagic vacuoles from endocytic/phagocytic compartments. In addition, It is difficult to assess and compare the size of autophagic vacuole. Another drawback of electron microscopy is its static nature, making it very difficult to follow what is really a highly dynamic process ${ }^{(34)}$.

Recent generation electron microscopy targets increasing resolution and insight into autophagosome formation in cells based on advances in cryo-EM tomography and focused ion beam-scanning electron microscopy (FIB-SEM) ${ }^{(35)}$. These techniques are likely to provide high resolution three-dimensional images of autophagic compartments that are free of artifacts caused by chemical fixation ${ }^{(36)}$. 
2. Immunofluorescence staining using primary antibodies for autophagy widely used markers as LC3B or P62 that are labeled with a fluorochrome. Within the double membrane resides a microtubuleassociated protein called light chain 3 (LC3), a mammalian homolog of yeast autophagy-related protein $8(\mathrm{Atg} 8)^{(37)}$. sequestosome1 (SQSTM1) or P62 is one of the best studied autophagy receptors involved in elimination of ubiqutinated protein aggregates and bacteria. the degree of autophagy can be assessed by enumeration of autophagosome formation by techniques such as laser scanning confocal microscopy as in figs 7 and $8^{(38)}$.

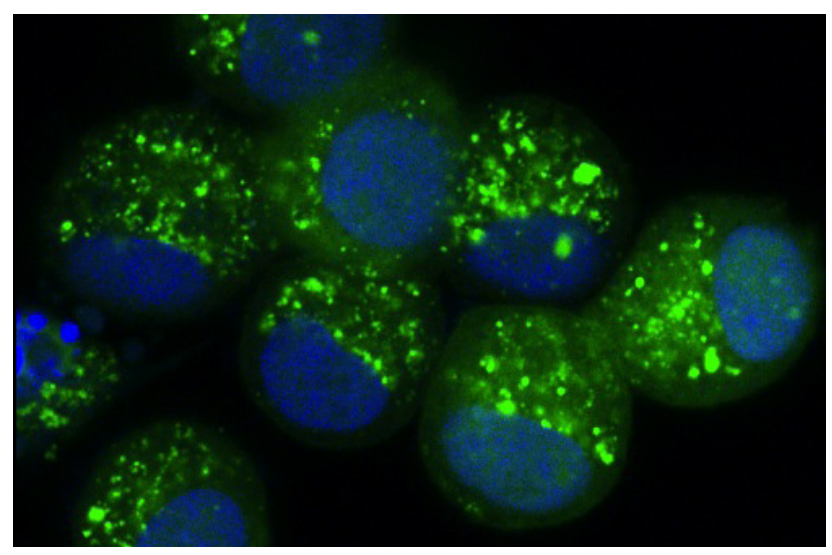

Fig. (7): Autophagosomes resident within the clustered cells and individual puncta are clearly visible in the cytoplasm of individual cells ${ }^{(39)}$.
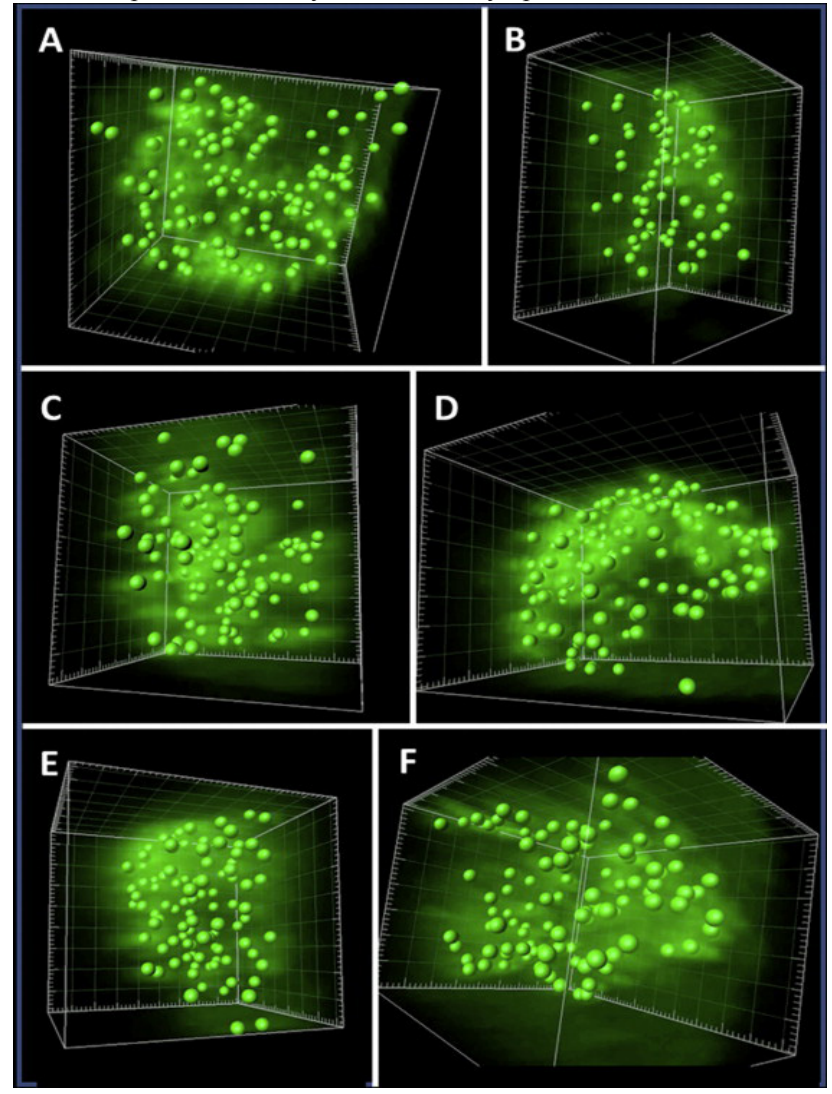

Fig. (8): Laser scanning confocal microscopy photomicrographs showing reconstruction of 3D images. Green signals represent LC3B protein in the membranes of autophagosomes ${ }^{(40)}$.
3. Immunohistochemistry using primary antibodies for autophagy markers as LC3B or P62 proved to be a reliable tool recruiting light microscopy ${ }^{(41)}$.

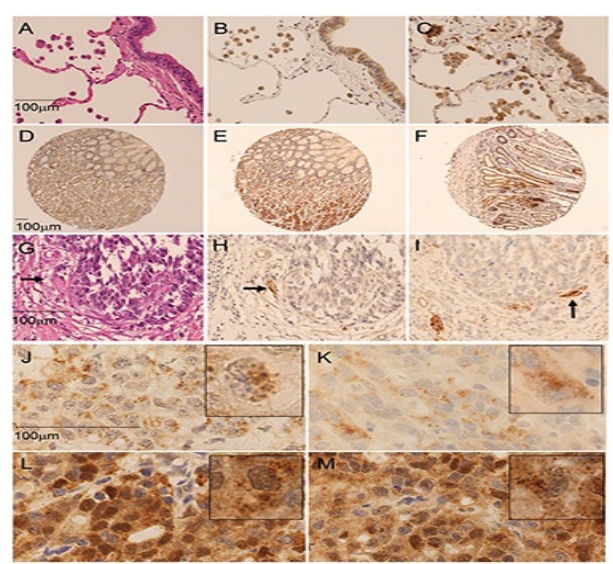

Fig. (9): Immunohistochemical staining for LC3B (J) and P62 (L) in lung carcinoma showing dot like pattern (puncta) of autophagy ${ }^{(42)}$.

4. Biochemical measurements such as the degradation of long-lived proteins as an indicator of autophagy activity are in use. This approach is based on the principle of radiolabeling longlived proteins with radioactive amino acids, such as [14C]-leucine, [3H]-leucine, [ 14C]-valine, or [35S]-methionine [31], before inducing autophagy and measuring the release of radioactivity from the labeled proteins, which reflects an activity of this pathway. Monodansylcadaverine (MDC) has been widely used as a specific marker for autophagic vacuoles, since it was shown to accumulate in acidic compartments enriched in lipids. A neutral derivative, monodansylpentane, was synthesized and this has been suggested to locate more exclusively into double-membraned structures ${ }^{(43)}$.

5. Western Blot-Based Assays for LC3 Lipidation. Currently, the analysis of LC3 lipidation by Western blot is the most appropriate method to assess changes in autophagy in most cell lines(43).

6. Flow Cytometry and Imaging Flow Cytometry Both techniques can be employed as a high-content analysis method to measure autophagic flux in living cells, especially if those do not adhere to surface, e.g., blood cells(43).
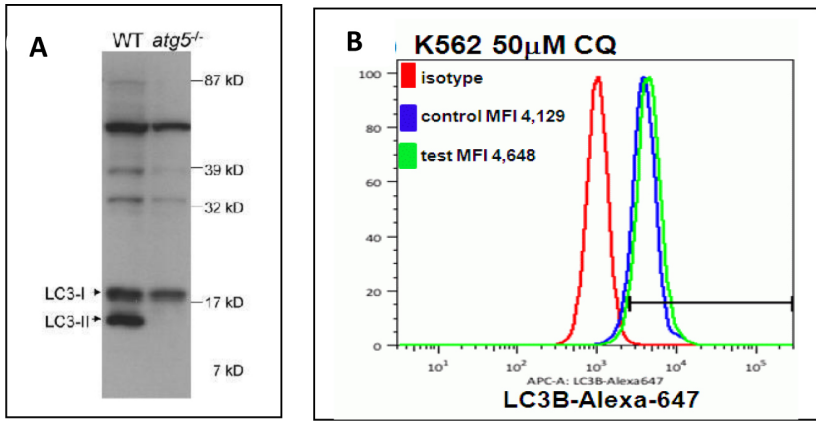

Fig. (10): Two charts for assessment of the autophagy marker LC3B using two different techniques: (A)Western blotting(44) and (B) flow cytometry $^{(45)}$. 


\section{AUTOPHAGY DYSFUNCTION}

Since autophagy dysfunction is linked to severe diseases such as neurodegeneration and cancer, the artificial control of autophagy promises to facilitate the development of therapeutic and preventive treatment for these severe diseases. Moreover, when point mutations that impair the formation of giant complex were introduced to $\operatorname{Atg} 13$, association of the autophagy initiation machinery was completely blocked ${ }^{(43)}$.

Autophagy occurs at a low basal level constitutively, and can be potently induced by various types of stress conditions, such as starvation, hypoxia, pathogen invasion, and exercise. Suppression of basal or stress-induced autophagy has been linked to a variety of pathological conditions, including tumorigenesis, neurodegeneration, inflammation, and metabolic diseases. Conversely, upregulation of autophagy has been suggested to be beneficial in preventing pathogenesis of many diseases. Hence, pharmacological strategies to activate autophagy have been recently studied, which were based on screens for autophagy inducers from small-molecule libraries or FDA-approved drug pools ${ }^{(46)}$.

Aging is a continuous physiological process affecting all the body cells and have a very strong impact on their performance. The homeostatic functions of autophagy with respect to turnover of long-lived proteins and removal of damaged organelles and cellular debris are believed to constitute an antiaging process ${ }^{(47)}$. Apparently, caloric restriction may represent a mechanism for reversing the age-dependent decline in autophagy ${ }^{(48)}$.

Neurodegenerative diseases are age-dependent hereditary or sporadic disorders that are manifested by progressive loss of neural function. Common features in their pathogenesis are mitochondrial dysfunction and the accumulation of protein aggregates as a result of mutation and impaired clearance mechanisms ${ }^{(49)}$. Autophagy is dysregulated in neurodegenerative disorders however, Pharmacologic stimulation of autophagy can alleviate symptoms related to neurodegeneration in mouse models $^{(50)}$

For example, in the brains of persons with Alzheimer's disease, the accumulation of autophagosomes containing hyperphosphorylated forms of microtubule-associated protein tau, leading to the formation of neurofibrillary tangles is accelerated ${ }^{(51)}$ or impaired autophagosomelysosome fusion ${ }^{(52)}$. Huntington's disease involve the accumulation of mutant proteins with polyglutamine-rich extensions due to autophagosomal cargo recognition or sequestration of essential autophagy proteins in mhtt aggregates ${ }^{(53)}$.

The involvement of mitochondrial dysfunction in neurodegeneration is exemplified by Parkinson's disease. where the mobilization of dysfunctional mitochondria to the autophagosome for turnover is impaired. Mutations in PINK1 and PARK2 (the genes that encode PINK1 and parkin, respectively) result in recessive familial forms of human Parkinson's disease and correlate with mitochondrial dysfunction in mouse models ${ }^{(54)}$. Thus, autophagy appears to represent an initial adaptive response in neurodegeneration, subject to inhibition by pathologic accumulation of substrates, which may represent a failed repair mechanism that also contributes to disease progression.

In case of infectious diseases, autophagy contributes to the regulation and function of innate and adaptive immune responses. Several medically important human pathogens are degraded in vitro by xenophagy, including bacteria (e.g., group A streptococcus, Mycobacterium tuberculosis, Shigella flexneri, Salmonella enterica, Listeria monocytogenes, and Francisella tularensis), viruses such as herpes simplex virus type 1 (HSV-1) and chikungunya virus, and parasites such as Toxoplasma gondii. Autophagy genes have been shown to play a protective role in vivo against many of these pathogens ${ }^{(55)}$.

It has been found that macrophage-specific deletion of Atg5 in mice increases their susceptibility to $M$. tuberculosis infection. Thus, pharmacologic up-regulation of autophagy, enhancement of strategies to target intracellular pathogens to autophagosomes, and inhibition of microbial virulence factors that block host autophagy defenses may represent novel strategies for the treatment of certain infectious diseases ${ }^{(46)}$. Notably, vitamin D and sirolimus (formerly called rapamycin) inhibit replication of the human immunodeficiency virus and of $\mathrm{M}$. tuberculosis in human macrophages through an autophagy-dependent mechanism. Furthermore, the antimycobacterial action of standard antituberculosis agents is associated with induction of autophagy ${ }^{(56)}$. Thus, the augmentation of autophagy-dependent adaptive immune responses may be beneficial in vaccine development. An increasing number of genetic links have been identified between autophagy genes and susceptibility to infectious and inflammatory diseases $^{(57)}$.

Divergent roles of autophagy have been reported in pulmonary diseases associated with declining lung function. Notably, increased autophagy was associated with a propathogenic and proapoptotic phenotype in chronic obstructive pulmonary disease (COPD), which results from chronic exposure to cigarette smoke. The expression of LC3B-II and autophagosome formation are increased in lung tissue from those patients. In animals subjected to long-term inhalation of cigarette smoke, a genetic deficiency in LC3B was associated with resistance to emphysema ${ }^{(58)}$. Mechanistic studies have linked increased autophagic activity with enhanced epithelial-cell apoptosis during exposure to cigarette smoke. However, alveolar macrophages isolated from human smokers without COPD showed evidence of impaired autophagic activity and the accumulation of substrates ${ }^{(59)}$. Genetic deficiency of $\alpha 1$-antitrypsin causes pulmonary emphysema and hepatic dysfunction, which are associated with pathologic accumulation of mutant $\alpha 1$-antitrypsin. Like other disorders of protein aggregation, autophagy may act 
as a clearance mechanism in this disease $\mathrm{e}^{(60)}$

Lung tissue isolated from patients with pulmonary hypertension, including those with idiopathic pulmonary arterial hypertension, have increased LC3B activation and autophagosome formation, as compared with lung tissue from patients without pulmonary vascular disease. A recent preclinical study suggests that impaired clearance of aggregated protein (aggrephagy) is the pathogenic mechanism in cystic fibrosis, a genetic disorder caused by mutation in the cystic fibrosis transmembrane conductance regulator (CFTR). Thus, therapeutic interventions aimed at correcting deficiencies in autophagy may be useful in these diseases $^{(61)}$.

Relatively little is known about the role of autophagy in asthma, which involves abnormal inflammatory responses of the airways to allergens. Increased autophagosomes have been noted in bronchial-biopsy specimens from patients with asthma, 15 and ATG5 expression is elevated in nasalbiopsy specimens from children with asthma.16 Intronic SNPs of ATG5 have been associated with an increased incidence of asthma and a decline in lung function in cohort studies of adults and with deterioration of lung function in children with asthma ${ }^{(62)}$.

Metabolic activity is also affected by autophagy as it regenerates and releases amino acids, lipids, and other metabolic precursors. Genetic deletion of autophagy proteins promotes the storage of triglycerides in lipid droplets in the liver, suggesting that autophagy acts as a regulator of lipid metabolism and storage. Specific mutations have been linked to Paget's disease, a disorder of bone metabolism ${ }^{(63)}$.

During exercise, autophagy is increased in cardiac and skeletal muscle, adipose tissue, and pancreatic beta cells. In mice, exercise-induced autophagy provides protection against glucose intolerance associated with a high-fat diet. Hepatic autophagy is down-regulated in the liver in mouse models of obesity and insulin resistance ${ }^{(64)}$. In contrast, adipose-specific deletion of autophagy proteins leads to altered homeostasis and differentiation of adipose tissue and promotes insulin sensitivity ${ }^{(65)}$.

Modulations in autophagy have been associated with diseases of the heart, including cardiomyopathies, cardiac hypertrophy, ischemic heart disease, heart failure, and ischemia-reperfusion injury ${ }^{(66)}$. Genetic X-linked deficiency in lysosome-associated membrane protein 2 (LAMP2), which assists in autophagosome-lysosome fusion, causes cardiomyopathy known as Danon's disease $^{(67)}$. In patients with this disease, cardiomyocytes with evidence of mitochondrial dysfunction have an increased number of autophagosomes, as does cardiac tissue from patients with heart failure. In a mouse model of desmin-related cardiomyopathy, autophagic activity was shown to provide cardioprotection ${ }^{(68)}$.

Experimental ischemia-reperfusion injury also causes morphologic indicators of autophagy to increase in response to stress signals, including depleted ATP, hypoxia, and altered $\mathrm{Ca} 2+$ balance and may play various roles, depending on the phase of the injury ${ }^{(69)}$. Increased numbers of autophagosomes are evident in macrophages from atherosclerotic plaques. Autophagy may stabilize atherosclerotic plaques by preventing macrophage apoptosis and plaque necrosis and by preserving efferocytosis $^{(70)}$.

\section{AUTOPHAGY AND CANCER}

Cancer develops when molecular pathways that control cellular proliferation and/or programmed cell death are subjected to genetic deregulations. The contribution of loss of type I apoptotic responses to cancer formation is now well established and has been extensively analyzed at the molecular level over recent years ${ }^{(71)}$. Interestingly, a tight correlation between reduced autophagy and cancer has been documented in the past, and recent work indicates that several proteins and pathways that are related to autophagy signaling are being deregulated during malignant transformation, resulting in reduced autophagic activity. In other words, the downregulation of autophagic activity in malignant cells suggests that a failure in autophagy signaling may be instrumental in cancer formation, and that autophagy may function under certain circumstances as a safeguard mechanism that restricts uncontrolled cell growth $^{(72)}$.

Yet, the mechanisms by which the reduced autophagy contributes to cancer etiology could be interpreted in different ways. They may reflect the advantage provided by the breakdown of type II autophagic cell death in cases where this process operates to eliminate cells when necessary ${ }^{(73)}$. Alternatively, reduced autophagy may increase the proliferative capacity of cells by different mechanisms. Another point to be considered with respect to autophagy/cancer relationship is the observation that some malignant cell types respond to anticancer agents by triggering autophagy, indicating the potential utility of autophagic cell death induction in cancer therapy ${ }^{(74)}$.

\section{AUTOPHAGY CONTROL; A NEW THERAPEUTIC HORIZON}

Current therapeutic targeting of autophagy in human disease is limited by an incomplete understanding of how the process contributes to pathogenesis, the lack of specificity of compounds that can influence autophagy, and the limited availability of candidate therapeutics with clinical efficacy. Pharmacologic enhancement of autophagy (i.e., with vitamin $\mathrm{D}$ or adenosine $5^{\prime}$-monophosphateactivated protein kinase [AMPK] activators) promises to benefit certain diseases ${ }^{(75)}$.

Current clinical trials are questioning the usefulness of autophagy as a target in disease. Chloroquine and its derivative, hydroxychloroquine, are being tested for enhancement of chemotherapeutic efficacy. The design of therapeutic agents is complicated by the fact that many autophagy proteins, as well as pharmacologic inhibitors including chloroquine, may also affect biologic processes independently of autophagy activation ${ }^{(76)}$. 
An improved understanding of the mechanisms by which autophagy can prevent pathogenesis may lead to the identification of new targets for both diagnostic and therapeutic purposes. Drug screening for agonists or antagonists of autophagic activity, including upstream regulators and downstream targets of autophagy, may yield additional therapeutic targets. If the advances in autophagy continue at an accelerated pace, agents acting on autophagy may eventually provide useful therapies for human diseases ${ }^{(77)}$

\section{REFERENCES}

1. de Duve C, Pressman B, Gianetto R, Wattiaux R and Appelmans F (1955). Tissue fractionation studies. 6. Intracellular distribution patterns of enzymes in rat liver tissue. Biochem J; 60: 604617.

2. Sabatini D and Adesnik M (2013). Christian de Duve: Explorer of the cell who discovered new organelles by using a centrifuge. Proc Natl Acad Sci U S A; 110 (33): 13234-13235.

3. Ashford TP and Porter KR (1962). Cytoplasmic components in hepatic cell lysosomes. J Cell Biol; 12: 198-202.

4. Takeshige K, Babe M, Tsuboi S, Noda T and Ohsumi Y (1992). Autophagy in yeast demonstrated with protease-deficient mutants and conditions for its induction. JCB; 119: 301-311.

5. Tsukada M and Ohsumi Y (1993). Isolation and characterization of autophagy-defective mutants of Saccharomyces cerevisiae. FEBS Lett; 333: 169-174.

6. Kuma A, Hatano M, Matsui M, Yamamoto A, Nakaya H, Yoshimori T, Ohsumi Y, Tokuhisa T, and Mizushima N (2004). The role of autophagy during the early neonatal starvation period. Nature; 432: 1032-1036.

7. Ohsumi Y (2014). Historical landmarks of autophagy research. Cell Res; 24: 9-23.

8. Choi AMK, Ryter SW and Levine B (2013). Autophagy in human health and disease. N Engl J Med; 368: 651-662.

9. "The Nobel Prize in Physiology or Medicine 2016". Nobelprize.org. Nobel Media AB 2014. Web. 21 Sep 2017. <http://www.nobelprize.org/ nobel_prizes/medicine/laureates/2016/>.

10. Fan Y, Wang N, Rocchi A, Zhang W, Vassar R, Zhou Y and He C (2017). Identification of natural products with neuronal and metabolic benefits through autophagy induction. Autophagy, 13 , Iss. 1 .

11. Deretic V. Autophagy in Immunity and Infection (2006). WILEY-VCH Verlag GmbH \& Co. KGaA, Weinheim ISBN: 31527-31450.
12. Clarke R, Cook K, Hu R, Facey C, Tavassoly I, Schwartz J, Baumann W, Tyson J, Xuan J, Wang Y, Wärri A and Shajahan AN (2012): Endoplasmic reticulum stress, the unfolded protein response, autophagy, and the integrated regulation of breast cancer cell fate. Cancer Res. 72(6), 1321-1331.

13. Mizushima N and Levine B (2010). Autophagy in mammalian development and differentiation. Nature Cell Biology; 12(9):823-830.

14. Salemi S, Yousefi S, Constantinescu MA, Fey MF and Simon HU (2012). Autophagy is required for self-renewal and differentiation of adult human stem cells.Cell Res; 22(2): 432-435.

15. Pan H, Cai N, Li M, Liu G-H, and Belmonte JK (2013). Autophagic control of cell 'stemness'. EMBO Mol Med; 5(3): 327-331.

16. Boya P, Reggiori F and Codogno P (2013). Emerging regulation and functions of autophagy. Nat Cell Biol; 15(7): 713-20

17. Huang J and Klionsky DJ (2007). Autophagy and human disease. Cell Cycle; 6: 1837-1849.

18. (https://cellbiology.med.unsw.edu.au/cellbiology/ index.php?title=File:Autophagy_types.jpeg\#file).

19. (https://theartofmed.wordpress.com/2015/05/24/ autophagy/).

20. Dunn Jr (1990). Studies on the mechanisms of autophagy: formation of the autophagic vacuole. $\mathrm{J}$ Cell Biol; 110:1923-1933.

21. Levine B (2005). Eating oneself and uninvited guests: autophagy-related pathways in cellular defense. Cell; 120: 159-162.

22. (https://www.sciencedirect.com/science/article/ pii/S0959440X16301488).

23. De Duve C (1966). Functions of lysosomes. Annu Rev Physiol; 28: 435-492.

24. Noda T, Suzuki K and Ohsumi Y (2002). Yeast autophagosomes: de novo formation of a membrane structure. Trends Cell. Biol.; 12: 231-235.

25. Mc Ewan D and Dikic I (2010). Not all autophagy membranes are created eaqualy. Cell; 141: 564-566.

26. Axe EL, Walker SA, Manifava M, Chandra P, Roderick HL, Habermann A, Griffiths G and Ktistakis NT (2008). Autophagosome formation from membrane compartments enriched in phosphatidylinositol 3-phosphate and dynamically connected to the endoplasmic reticulum. J Cell Biol; 182: 685-701.

27. Lamb C A, Yoshimori T and Tooze S A (2013). The autophagosome: origins unknown, biogenesis complex. Nature Reviews Molecular Cell Biology; 14: 759-774. 
28. Kerr JF, Wyllie AH and Currie AR (1972). Apoptosis: a basic biological phenomenon with wide-ranging implications in tissue kinetics. Br. J. Cancer; 26: 239-257.

29. Clarke PG (1990). Developmental cell death: morphological diversity and multiple mechanisms. Anat. Embryol. (Berlin); 181: 195-213.

30. Gozuacik D and Kimchi A (2004). Autophagy as a cell death and tumor suppressor mechanism. Oncogene; 23: 2891-2906.

31. Chiara Maiuri M, Zalckvar E, Kimchi A and Kroemer G (2007). Self-eating and self-killing: crosstalk between autophagy and apoptosis Nature Rev. Mol. Cell Biol., 8: 741-752.

32. Arstila AU, and Trump BF (1968). Studies on cellular autophagocytosis. The formation of autophagic vacuoles in the liver after glucagon administration. Am J Pathol; 53: 687-733.

33. http://www.med.monash.edu.au/biochem/docs/ lazarou-figure $1 \mathrm{sml} . j p g$.

34. Pfeifer U (1987). Functional morphology of the lysosomal apparatus. In: Glaumann $\mathrm{H}$, Ballard FJ,eds. Lysosomes: Their role in protein breakdown. London: Academic Press,:3-59.

35. Hurley JH and Nogales E (2016). Next-generation electron microscopy in autophagy research. Curr Opin Struct Biol; 41:211-216.

36. Ylä-Anttila $P$, Vihinen $H$, Jokitalo E and Eskelinen EL (2009). Monitoring Autophagy by Electron Microscopy in Mammalian Cells. Meth in Enzym; (452): 143-164.

37. Kabeya Y, Mizushima N, Ueno T, Yamamoto A, Kirisako T, Noda T, Kominami E, Ohsumi Y, and Yoshimori T (2000). LC3, a mammalian homologue of yeast Apg8p, is localized in autophagosome membranes after processing. EMBO J; 19: 5720-5728.

38. Klionsky DJ, Abdalla FC, Abeliovich H, Abraham RT, Acevedo-Arozena A, Adeli K, et al. (2012). Guidelines for the use and interpretation of assays for monitoring autophagy. Autophagy ;8:445-544.

39. http://www.bcgsc.ca/faculty/sgorski/gfpautolysozomes/image_view_fullscreen.

40. Jackson W, Yamada M, Moninger T and Grose C (2013). Visualization and quantitation of abundant macroautophagy in virus-infected cells by confocal three-dimensional fluorescence imaging. J Virol Meth; 193(1): 244-250.

41. Orhon I and Reggiori F (2017). Assays to Monitor Autophagy Progression in Cell Cultures. Cells; 6: 20-28.
42. Schläfli A M, Berezowska S, Adams O, Langer $\mathrm{R}$ and Tschan M P (2015). Reliable LC3 and P62 autophagy marker detection in formalin fixed paraffin embeded human tissue by immunohistochemistry. Europian Journal of Histochemistry, Technical note, DOI: 10.4081/ ejh.2015.248/.

43. Behrends C, Sowa, ME, Gygi SP and Harper JW (2010). Network organization of the human autophagy system. Nature; 466(7302): 68-76.

44. https://images.novusbio.com/images $2 / L C 3 \% 20$ Antibody\%20Pack-Western\%20Blot-NB91040435-img0007.jpg.

45. http://www.icms.qmul.ac.uk/flowcytometry/uses/ autophagy/diagrams/rapa.gif.

46. Rubinsztein DC, Codogno P and Levine B (2012). Autophagy modulation as a potential therapeutic target for diverse diseases. Nat Rev Drug Discov; 11(9): 709-730.

47. Rubinsztein DC, Mario G and Kroemer G (2011). Autophagy and aging. Cell;146: 682-695.

48. Jia K and Levine B (2007). Autophagy is required for dietary restriction-mediated life span extension in C. elegans. Autophagy; 3: 597-599.

49. Jellinger KA (2010). Basic mechanisms of neurodegeneration: a critical update. J Cell Mol Med;14:457-487.

50. Schaeffer V, Lavenir I, Ozcelik S, Tolnay M, Winkler DT, Goedert M (2012). Stimulation of autophagy reduces neurodegeneration in a mouse model of human tauopathy. Brain; 135: 2169-77.

51. Ma JF, Huang Y, Chen SD, Halliday G (2010). Immunohistochemical evidence for macroautophagy in neurones and endothelial cells in Alzheimer's disease. Neuropathol Appl Neurobiol; 36: 312-319.

52. Uddin MS, Stachowiak A, Mamun AA, Tzvetkov NT, Takeda S, Atanasov AG, Bergantin LB, AbdelDaim MM and Stankiewicz AM (2018). Autophagy and Alzheimer's Disease: From Molecular Mechanisms to Therapeutic Implications. Front Aging Neurosci.; 30 (10):04. doi:10.3389/ fnagi.2018.00004.

53. Shibata M, Lu T, Furuya T, et al (2006). Regulation of intracellular accumulation of mutant Huntingtin by Beclin 1. J Biol Chem; 28:14474-14485.

54. Trancikova A, Tsika E and Moore DJ (2012). Mitochondrial dysfunction in genetic animal models of Parkinson's disease. Antioxid Redox Signal; 16: 896-919. 
55. Levine B, Mizushima N and Virgin HW (2011). Autophagy in immunity and inflammation. Nature; 469: 323-335.

56. Campbell GR and Spector SA (2011). Hormonally active vitamin D3 (1alpha,25dihydroxycholecalciferol) triggers autophagy in human macrophages that inhibits HIV-1 infection. J Biol Chem; 286: 18890-18902.

57. Cucu MG, Streața L, Lelia Riza A, Cimpoeru AL, Şerban-Şoşoi S, et al. (2017). Polymorphisms in autophagy genes and active pulmonary tuberculosis susceptibility in Romania. Revista Română de Medicină de Laborator; 25(1): 47-53.

58. Chen ZH, Kim HP, Sciurba FC, et al. (2008). Egr1 regulates autophagy in cigarette smoke-induced chronic obstructive pulmonary disease. PLoS One; 3(10): 3316-3323.

59. Monick MM, Powers LS, Walters K, et al. (2010). Identification of an autophagy defect in smokers' alveolar macrophages. J Immunol; 185: 5425-5435.

60. Teckman JH, An JK, Blomenkamp K, Schmidt B and Perlmutter D (2004). Mitochondrial autophagy and injury in the liver in alpha 1-antitrypsin deficiency. Am J Physiol Gastrointest Liver Physiol; 286: G851-G562.

61. Lee SJ, Smith A, Guo L, et al (2011). Autophagic protein LC3B confers resistance against hypoxiainduced pulmonary hypertension. Am J Respir Crit Care Med;183: 649-658.

62. Martin LJ, Gupta J, Jyothula SS, et al. (2012). Functional variant in the autophagy-related 5 gene promotor is associated with childhood asthma. PLoS One; 7(4): e33454.

63. Singh R, Kaushik S, Wang Y, et al. (2009). Autophagy regulates lipid metabolism. Nature; 458: 1131-1135.

64. Yang L, Li P, Fu S, Calay ES and Hotamisligil GS (2010). Defective hepatic autophagy in obesity promotes ER stress and causes insulin resistance. Cell Metab;11: 467-78.

65. Singh R, Xiang Y, Wang Y, et al. (2009). Autophagy regulates adipose mass and differentiation in mice. J Clin Invest; 119: 3329-3339.

66. Kirshenbaum LA (2012). Regulation of autophagy in the heart in health and disease. J Cardiovasc Pharmacol;60:109-116.
67. Nishino I, Fu J, Tanji K, et al.(2000). Primary LAMP-2 deficiency causes X-linked vacuo-lar cardiomyopathy and myopathy (Danon disease). Nature; 406: 906-910.

68. Tannous P, Zhu H, Johnstone JL, et al. (2008). Autophagy is an adaptive response in desminrelated cardiomyopathy. Proc Natl Acad Sci U S A 2008;105:9745-9750.

69. Lin XL, Xiao WJ, Xiao LL, Liu MH (2018). Molecular mechanisms of autophagy in cardiac ischemia/reperfusion injury (Review). Mol Med Rep;18(1): 675-683.

70. Liao X, Sluimer JC, Wang Y, et al. (2012). Macrophage autophagy plays a protective role in advanced atherosclerosis. Cell Metab;15: 545-553.

71. Tait SW and Green DR (2008). Caspaseindependent cell death: leaving the set without the final cut. Oncogene; 27: 6452-6461.

72. Hanahan D and Weinberg RA (2011). Hallmarks of cancer: the next generation. Cell; 144(5): 64674.

73. Abraham NM, Kirubel MM and Abraham DA (2018). Autophagy as a Possible Target for Cancer Therapy. J Orthop Oncol, 4:2 DOI: 10.4172/2472016X.1000124.

74. Marinković $M$, Šprung $M$, Buljubašić $M$ and Novak I (2018). Autophagy Modulation in Cancer: Current Knowledge on Action and Therapy. Oxidative Medicine and Cellular Longevity; Volume 2018, Article ID 8023821, 18 pages. doi. org/10.1155/2018/8023821.

75. Dai J, Liang Y, Li H, Zhou W, Wang B, Gong A and Zhang R (2018). Vitamin D enhances resistance to aspergillus fumigatus in mice via inhibition of excessive autophagy. Am J Transl Res; 10(2): 381-391.

76. Gao L, Sun X, Zhang Q, Chen X, Zhao T, Lu L, Zhang J, Hong Y (2018). Histone deacetylase inhibitor trichostatin A and autophagy inhibitor chloroquine synergistically exert anti-tumor activity in H-ras transformed breast epithelial cells. Mol Med Rep; 17(3): 4345-4350.

77. Cheng Y, Ren X, William N. Hait and Yang J (2013). Therapeutic Targeting of Autophagy in Disease: Biology and Pharmacology. Pharmacol Rev; 65(4): 1162-1197. 